\title{
La gammaglutamil transpeptidasa en el recién nacido
}

\author{
Dr. Víctor Casanueya E.1; Matr. Ana M. Monsalves G. ${ }^{2}$; \\ BQ Luis Rifo F. ${ }^{3}$ \\ Serum gamma glutamil transferase in \\ newborn infants
}

\begin{abstract}
Serum gamma glutamil transpeptidase activity (SGGTA) was measured in 83 term neonates along the first week of life. Mean SGGTA at birth were 36.8 U/L (range: 23-81 U/L). At fourth and seventh day of life, SGGTA values were $42 \mathrm{U} / \mathrm{L}$ (range: 12-116 U/L) and $46 \mathrm{U} / \mathrm{L}$ (range: 12-116 U/L) respectively. Maternal SGGTA among 15 pregnant women were $12.87 \mathrm{U} / \mathrm{L}$ (range: 5-27 U/L), well bellow the normal upper linit of $34 \mathrm{U} / \mathrm{L}$ and significantly lower than those from their own newborn babies in the first week of life ( $\mathrm{p}<0.001$ ). The source and significance of the increased enzyme activity in term neonates is uncertain. No correlations were found between individual serum bilirrubin on day seven and SGGTA in venous and cord blood samples from the first hours of life.

(Key words: serum gamma glutamil transpeptidase activity; cord blood, neonatal, maternal, serum bilirrubin.)
\end{abstract}

La gammaglutamil transpeptidasa (GGT) es una enzima que se determina con frecuencia en clinica, cuya actividad aumenta en forma inespecifica, entre otras, en afecciones hepatobiliares acompañadas de ictericia. A diferencia de las fosfatasas alcalinas no aumenta en el embarazo, lo que le da cierto valor en el diagnóstico de aquéllas ${ }^{1-3}$.

En el pais sólo se ha comunicado estudios de su actividad en adultos ${ }^{4}$. Por otra parte, en casi todos los recién nacidos ocurren aumentos de la bilimubinemias, que pueden tener serias consecuencias si son intensos.

La inmadurez en la actividad de la glucuronil transferasa, encargada de conjugar la bilirrubina, origina la llamada "ictericia fisiológica en el recién nacido". La medición de esta enzima es dificil en forma rutinaria, pues implica hacer biopsia hepática ${ }^{6}$, razón por la cual se han buscado otros índices de su actividad que alerten al eventual aumento de la bilirrubinemia. Según Davidson la GGT podría ser útil en ese sentido?.

El propósito de este estudio fue determinar los valores de referencia de GGT en una po-

1. Departamento de Pediatría, Facultad de Medicina, Universidad de Concepción.

2. Departamento de Obstetricia y Puericultura, Facultad de Medicina, Universidad de Concepción.

3. Departamento de Bioquímica Aplicada, Facultad de Farmacia, Lniversidad de Concepción. blación de recién nacidos de término normales; establecer si guardan relación con la actividad enzimática de las madres respectivas y con la evolución de la bilirrubinemia en las primeras semanas de vida.

\section{MATERJAL Y METODO}

Esta investigación se realizó en el Hospital Guillermo Grant Benavente de Concepción (laboratorio central, unidad de recién nacidos y banco de sangre) y además eI Departamento de Bioquírnica Aplicada de la Facultad de Farmacia de la Universidad de Concepción.

En una población de 84 recién nacidos normales se estudió la actividał de la GGT, junto con los niveles de bilirrubinemia al nacer, al cuarto y séptimo días de vida. En un grupo de 15 recién nacidos se estudió en forma simultánea la actividad de GGT y ta de las madres respectivas en el momento del parto. Para las mediciones de GGT se usaron juegos de "monotest a", Boebringes Mannhein, que emplean el método de Szasz y $\mathrm{col}^{8}$. Los recién nacidos fueron examinados por médicos del Departamento de Pediatría de la Facultad de Medicina, y sus madres, por médicos del Servicio de Obstetricia.

Las madres habjan recibido atención prenatal por matronas y obstetras; ningtuna presentó complicaciones durante la gestación. El parto fue por vía raginal en todos los casos, sin inducción con ocitocina.

Los recién nacidos fueron considerados normales en el examen físico inicial, con Apgar en uno y cínco minutos sobre 7 y edad gestacional mayor de 38 semanas. Se excluyeron los nin̄os con potencial incompatibilidad de grupos ABO y Rh, tambiér los hijos de madres que estuvieran recibiendo fenobarbital, pues la droga influye sobre la actividad de GGT ${ }^{9,10}$. 
Las muestras de sangre fueron obtenidas por la misma matrona de la fontanela anterior hasta, máximo, cinco horas después del nacintento.

Las determinaciones de bilirrubina se hicieron segün el método de Malloy y Evelyn modifjcadol. Los resultados se analizaron mediante prueba " $t$ " de Student $y$ el método de correlación de Kendall.

\section{RESULTADOS}

Inicialmente se estudió la precisión del método de Szasz con muestras de sangre procedentes de 20 personas normales y de 21 enfermos con actividad GGT elevada. En ambos grupos se obtuvo un coeficiente de variación de $3,3 \%$ que demuestra buena precisión. También se estudió la precisión en el tiempo, haciendo 20 determinaciones en días alternos, encontrándose un rango de variación, respecto al promedio, de $25 \mathrm{U} / \mathrm{L}$, cuyo coeficiente también resultó $3,3 \%$.

La distribución de la actividad de la GGT en las madres fue, en promedio, $12,87 \mathrm{U} / \mathrm{L}$, rango 5 a $17 \mathrm{U} / \mathrm{L}$.

En los recién nacidos las actividades promedio de GGT fueron en el primer día de vida 36,8 $\mathrm{U} / \mathrm{L}$, rango 23 a $81 \mathrm{U} / \mathrm{L}$; en el cuarto día de vida $42,3 \mathrm{U} / \mathrm{L}$, rango 12 a $116 \mathrm{U} / \mathrm{L}$ y en el séptimo dia $46,8 \mathrm{U} / \mathrm{L}$, rango 12 a $116 \mathrm{U} / \mathrm{L}$ (figura 1 ).

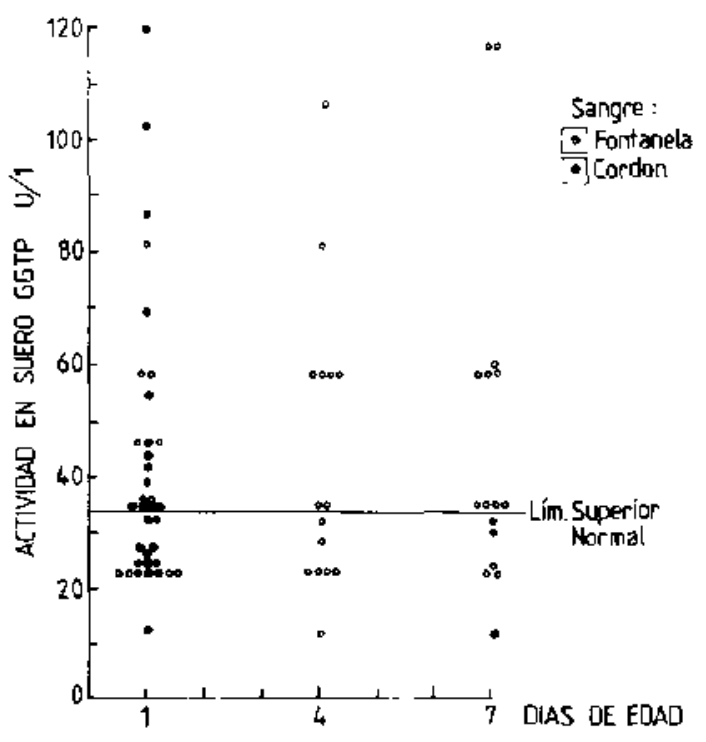

Figura 1: Actividad de GGT en suero de 67 RNT en la primera semana de vida.
La actividad de GGT en las madres fue significativamente más baja que en los recién nacidos durante la primera semana de vida $(\mathrm{p}<0,001)$. No se encontró correlación entre los valores individuales de GGT de cada madre con su hijo.

No se encontraron diferencias significativas en Ia actividad de GGT de los recién nacidos en los días primero, cuarto y séptimo de vida.

Para verificar si es posible usar la actividad de GGT al nacer para predecir hiperbilirrubinemia posterior, se calculó el coeficiente de correlación entre dicha actividad en el primer día y la bilirrubinemia total en el 70 día de vida, lo que dio un valor de $\mathrm{r}^{2}=0,02$, que no es significativo (tabla I).

Dado que la actividad de GGT podría ser mayor en la sangre del cordón umbilical ${ }^{7}$ se calculó también el coeficiente de correlación entre actividad de GGT en muestras de sangre de cordón y bilirrubinemia del séptimo día en 22 recién nacidos, resultando, como en el caso anterior, un valor de $t^{2}=0,01$, que no es significativo (tabla 2).

\section{DISCUSION}

La distribución de la actividad de GGT ottenida en las madres de este estudio resultó igual a la de mujeres sanas no embarazadas cuyo promedio fue $12,87 \mathrm{U} / \mathrm{L}$, rango 5 de $27 \mathrm{C} / \mathrm{L}^{12}$.

Tabla 1

Actividad de GGT al nacer y bilirrubinemia al $7^{\circ}$ día de vida, (Sangre de fontanela). En 15 recién aacidos

\begin{tabular}{rcc}
\hline RN & $\begin{array}{c}\text { Actividad } \\
\text { GGT }(\mathbf{U} / \mathrm{L})\end{array}$ & $\begin{array}{c}\text { Bilirrubinemia } \\
\text { 70 dia (mg \%) }\end{array}$ \\
\hline 1 & 23 & 2,41 \\
2 & 35 & 2,20 \\
3 & 23 & 3,85 \\
4 & 81 & 5,16 \\
5 & 46 & 4,97 \\
6 & 58 & 3,20 \\
7 & 35 & 9,25 \\
8 & 12 & 5,40 \\
9 & 58 & 3,71 \\
10 & 46 & 8,03 \\
11 & 23 & 3,41 \\
12 & 24 & 6,93 \\
13 & 24 & 2,17 \\
14 & 32 & 1,67 \\
15 & 23 & 2,86 \\
\hline
\end{tabular}


Tabla 2

Actjyidad de GGT al nacer y bilirrubinemia a $7^{\circ} \mathrm{d}$ ia. (Sangre del cordón umbilical). En 22 recién nacidos

\begin{tabular}{rcr}
\hline RN & $\begin{array}{c}\text { Actividad } \\
\text { GGT (U/L) }\end{array}$ & $\begin{array}{c}\text { Bilirrubinemia } \\
70 \text { día (mg/dL) }\end{array}$ \\
\hline 1 & 44 & 8,35 \\
2 & 35 & 7,32 \\
3 & 87 & 1,63 \\
4 & 35 & 6,22 \\
5 & 55 & 0,72 \\
6 & 42 & 9,77 \\
7 & 13 & 10,04 \\
8 & 23 & 6,02 \\
9 & 35 & 12,42 \\
10 & 39 & 2,23 \\
11 & 28 & 2,80 \\
12 & 120 & 7,81 \\
13 & 23 & 2,70 \\
14 & 33 & 14,04 \\
15 & 23 & 1,23 \\
16 & 28 & 6,82 \\
17 & 46 & 11,10 \\
18 & 27 & 8,74 \\
19 & 103 & 16,14 \\
20 & 70 & 1,36 \\
21 & 26 & 9,45 \\
22 & 35 & 2,96 \\
\hline
\end{tabular}

Se considera que el límite superior normal de actividad GGT es $34 \mathrm{U} / \mathrm{L}^{7}, 12,14$. La actividad demostrada en sangre de fontanela coincide con lo publicado en 82 RNT de 9 horas a 11 dias de edad, cuyo promedio fue $46 \mathrm{U} / \mathrm{L}$, rango 4 a $26 \mathrm{~J}$ U/L. En la misma serie tampoco se encontró relación entre la actividad de GGT en madres e hijos, lo que hace improbable el paso transpla. centario de la enzima desde la madre al feto ${ }^{15}$.

Contrariamente a lo sostenido por otros au. tores $^{2,16}$, en recién nacidos la actividad GGT es mayor que en edades posteriores. La explicación de este hecho no es clara: se sabe que en el adulto la GGT interviene en los procesos de absorción y secreción, pues se encuentra en altas concentraciones en células dotadas con microvellosidades (p.e. riñón y canalículos biliares): la GGT facilita el transporte de aminoácidos a través de las membranas celulares utilizando glutation $^{17}$. Por otra parte el glutation es también usado para desintoxicar, produciéndose en el proceso ácido mercaptúrico que es a su vez catabolizado por la GGT. Se piensa que, cuando el hígado es inmaduro, los mecanismos enzimáticos de síntesis y utilización de glutation se adaptan para desintoxicar : como ocurriría en los reçién nacidos $^{1 ?}$.

Otros autores han estudiado la diferencia arterio venosa de actividad de GGT en los vasos umbilicales, encontrando el máximo en las venas, lo que sugiere que la placenta es el lugar de origen. También es alta la actividad en la membrana de amnios y en las vellosidades placenteras, siendo improbable el origen fetal, pues no observa su aumento en casos con atresia duodenal ${ }^{20}$.

También se ha postulado que la GGT puede proceder de otros tejidos: mediante electroforesis se han separado dos isoenzimas ${ }^{21}$ y se han descrito dos tipos (tetal y adulto) diferentes en la conposición de la glicoproteínas.

Sobre la base de nuestros resultados la determinación de la GGT no es útil para el diagnóstico anticipado de hiperbilirrubinemia en recién nacidos. No se pudo comprobar la correlación encontrada por Davidson ${ }^{7}$, que podría haber sido influida por el escaso número de sujetos de su muestra. Por lo demás, sus estudios en sangre de cordón en los días cuarto y séptimo son de improbable factibilidad en la prạctica.

Nuevas líneas de investigación se han desarrollado sobre la GGT en genética y perinatología: en Francia se está estudiando su actividad en el liquido amniótico de fetos con anomalías cromosómicas $^{22}$ y la relación entre niveles maternos de GGT y la presencia de anomalias en el recién nacido que permitan la identificación precoz del síndrome fetal alcohólico ${ }^{23}$.

\section{RESUMEN}

La actividad de la gammaglutamil transpeptjdasa (GGT) fue determinada en 83 recién nacidos de término normales durante la primera semana de la vida. Las actividades promedio fueron: 36,8 U/L en el primer día, rango 23 a $81 \mathrm{U} / \mathrm{L} ; 42$, $\mathrm{U} / \mathrm{L}$, rango de 12 is $116 \mathrm{U} / \mathrm{L}$ en el cuarto dia $\mathrm{y}$ $46,8 \mathrm{U} / \mathrm{L}$, rango 12 a $116 \mathrm{U} / \mathrm{L}$ en el séptimo día de vida. La actividad materna promedio en 15 embarazadas fue $12,87 \mathrm{U} / \mathrm{L}$, rango 5 a $27 \mathrm{U} / \mathrm{L}$, inferior a $34 \mathrm{U} / \mathrm{L}$ (límite superior normal). Estas cifras son similares a las encontradas en mujeres normales. La actividad en las madres fue significativamente menor que en los recién nacidos ( $<<0,001)$. La razôn de la mayor actividad en los hijos no ha sido explicada. No se encontró relación entre los valores de GGT y de bilirrubi. 
nemia en la primera semana, por lo cual se descarta su valor predictivo de hiperbilirrubinemia.

\section{REFERENCIAS}

1. Lum G, afd Gampinor B.: Scrum g-Glutamyl transpeptidase activity as an indicator of disease of liver, pancreas or bone. Clin Chem 1972; 18 : 358-361.

2. Boone D., Routh J., Schrontz R.: G-Glutanyl txanspeptidase and 5 Nucleotidase. Am J Clin Pathol 1974; 61: 321-323.

3. Burrows $S$. Feldman $W$. , Me Bride F.: Serum g-Glutamy1 transpeptidase. Evaluation in screening of hospitalized patients. Am J Clin Pathol 1975; 64: $311-315$.

4. Sirón $M_{1}$ Castillo $T$, Welff $C$. Soto $J .:$ La Gana Glutamil transpeptidasa en la práctica clínica. Rey Med Chile 1980; 108: 332-337.

5. Maisels $f_{\text {.: }}$ Jaundice in the newborn. Ped in Revicw 1982: 3: 305-319.

6. Casantueva $\boldsymbol{V}$. Hìperbilirrubinemia no hemolítica congénita. Rev Chil Pediatr 1983; 54 : 344-349.

7. Davidson D. Mc Intosh $W$, and Ford $J$ : Cord g-Glutamyl transpeptidase activity and neonatal jaundice. Atch Dis Chil 1976; 51: 286-288.

8. Sizasz. G.A.- Kinetic photometric method for serum g-Glutan1yl transpeptidase. Clin Chem 1969; 15: $124-127$.

9. Whitfield J.B., Pounder R.E., Neale G.: Serum g-Glutamyl transpeptidase activities in liver disease. Gut 1972; 13: 702-708.

10. Schmidr, f,: G-Glutamyl transpeptidase. Dtsch Med Wschr 1973;98: 1572-1578.

11. Todd-Sanford: Diagnóstico Clínico por el Laboratorio. 60 Ed. Salvat 1979: 616-617.

12. Walker, F.B.; Hoblit, D.L. and Combes B.. G-Glutamyl transpeptidase in normal pregnancy Obstet Gynecol 1974:43: 745-749.
13. Cohen $M$. and $M C$ Namara $H$.: The diagnostic value of gamma Glutamyl transpeptidase in children and adolescents with diseases. J Pediatr 1969; 75: 5-7.

14. Richterich $R$, and Cant Z.R.: Values of plasma garnmaglutamyl transpeptidase en clildren. Enzymol 1972; 13: 257*260.

15. Shore G.M., Hoberman L., Ben A., Dowdey C. and Burton Combes: Serum gamma glutamyl transpeptidasc activity in normal children. Am J Clin Pathol 1975; 63: 245-250.

16. Goldbarg J., Pinteda $E_{\text {., Smith }} E_{\text {. }}$, Friedmann $O$, Rutenbuerg A.: A method for the colorimetric determination of g-Glutamyl transpeptidase in human serum: enzimatic activity in health and disease, Gastroenterol 1963: 44: 127-129.

17. Merster $A$.: On the enzymology of amino acid transport. Science 1972;180:33-39.

18. Hones G., Hird F, and Isherword F.A.: Enzymatic transpeptidation reactions involving GOT and amino acyl peptides. Biochem I 1952;51:25-35.

19. Prislsc $A$. and Didala $A$ : Variation of the serum GGT activity in full term and pretern babjes during their first 2 week of like. Min Pediatr 1980 32: 291-292.

20. Kötgen $E$, Reutter $W$. and Gerok $w$.: Different GGT during development of liver and small intestine. Biochem Biophys Res Commun 1976; 72 : $61-64$.

21. Nemesansky $E$, and Lott A,J.: GGT and isoenzymes. Clin Chem 1985; 31: 797-803.

22. Muller $F_{.,}$Rebiffé M., Der Sarkissiantt, Bové J., Bové $A$.: Decrease of various enzymes activities in the amnotic fluid of the fetuses with chiomosonal anomalies. Ann Genet (Paris) 1986; 29:27-31.

23. Halpérin D.S., Assimacopoulos A., Lacourt $G, F$. Béquin, Ferrier P.E.: GGT sérique maternelle et dépistage prenatal de syndrome de l'alcoolisme fetal. J Gynecol Obstet Biol Reprod 1986; 15: 165-170. 